\title{
THE BEST CONSTANT IN A WEIGHTED HARDY-LITTLEWOOD-SOBOLEV INEQUALITY
}

\author{
WENXIONG CHEN AND CONGMING LI
}

(Communicated by David S. Tartakoff)

Abstract. We prove the uniqueness for the solutions of the singular nonlinear PDE system:

$$
\left\{\begin{array}{l}
-\triangle\left(|x|^{\alpha} u(x)\right)=\frac{v^{q}(x)}{|x|^{\beta}}, \\
-\triangle\left(|x|^{\beta} v(x)\right)=\frac{u^{p}(x)}{|x|^{\alpha}} .
\end{array}\right.
$$

In the special case when $\alpha=\beta$ and $p=q$, we classify all the solutions and thus obtain the best constant in the corresponding weighted Hardy-LittlewoodSobolev inequality.

\section{INTRODUCTION}

Let $0<\lambda<n, 1<s, r<\infty$, and $\|f\|_{p}$ be the $L^{p}\left(R^{n}\right)$ norm of the function $f$. The well-known classical Hardy-Littlewood-Sobolev inequality (HLS) states that:

$$
\int_{R^{n}} \int_{R^{n}} \frac{f(x) g(y)}{|x-y|^{\lambda}} d x d y \leq C_{s, \lambda, n}\|f\|_{r}\|g\|_{s}
$$

for any $f \in L^{r}\left(R^{n}\right), g \in L^{s}\left(R^{n}\right)$, and for $\frac{1}{r}+\frac{1}{s}+\frac{\lambda}{n}=2$.

Hardy and Littlewood also introduced the double weighted inequality, which was later generalized by Stein and Weiss in [13]. It reads:

$$
\left|\int_{R^{n}} \int_{R^{n}} \frac{f(x) g(y)}{|x|^{\alpha}|x-y|^{\lambda}|y|^{\beta}} d x d y\right| \leq C_{\alpha, \beta, s, \lambda, n}\|f\|_{r}\|g\|_{s}
$$

where $\alpha+\beta \geq 0$,

$$
1-\frac{1}{r}-\frac{\lambda}{n}<\frac{\alpha}{n}<1-\frac{1}{r}, \text { and } \frac{1}{r}+\frac{1}{s}+\frac{\lambda+\alpha+\beta}{n}=2 .
$$

To obtain the best constant in the weighted inequality (3), one can maximize the functional

$$
J(f, g)=\int_{R^{n}} \int_{R^{n}} \frac{f(x) g(y)}{|x|^{\alpha}|x-y|^{\lambda}|y|^{\beta}} d x d y
$$

Received by the editors November 13, 2006.

2000 Mathematics Subject Classification. Primary 35J45, 35J60; Secondary 45G05, 45 G15.

Key words and phrases. Weighted Hardy-Littlewood-Sobolev inequality, best constants, system of singular PDEs, uniqueness, radial symmetry, classifications.

The first author was partially supported by NSF Grant DMS-0604638.

The second author was partially supported by NSF Grant DMS-0401174. 
under the constraints $\|f\|_{r}=\|g\|_{s}=1$. The corresponding Euler-Lagrange equations are the system of integral equations:

$$
\left\{\begin{array}{l}
\lambda_{1} r f(x)^{r-1}=\frac{1}{|x|^{\alpha}} \int_{R^{n}} \frac{g(y)}{|y|^{\beta}|x-y|^{\lambda}} d y, \\
\lambda_{2} s g(x)^{s-1}=\frac{1}{|x|^{\beta}} \int_{R^{n}} \frac{f(y)}{|y|^{\alpha}|x-y|^{\lambda}} d y,
\end{array}\right.
$$

where $f, g \geq 0, x \in R^{n}$, and $\lambda_{1} r=\lambda_{2} s=J(f, g)$.

For $u=c_{1} f^{r-1}, v=c_{2} g^{s-1}, p=\frac{1}{r-1}, q=\frac{1}{s-1}, p q \neq 1$, and for a proper choice of constants $c_{1}$ and $c_{2}$, system (6) becomes

$$
\left\{\begin{array}{l}
u(x)=\frac{1}{|x|^{\alpha}} \int_{R^{n}} \frac{v(y)^{q}}{|y|^{\beta}|x-y|^{\lambda}} d y, \\
v(x)=\frac{1}{|x|^{\beta}} \int_{R^{n}} \frac{u(y)^{p}}{|y|^{\alpha}|x-y|^{\lambda}} d y,
\end{array}\right.
$$

where $u, v \geq 0,0<p, q<\infty, 0<\lambda<n, \frac{\alpha}{n}<\frac{1}{p+1}<\frac{\lambda+\alpha}{n}$, and $\frac{1}{p+1}+\frac{1}{q+1}=\frac{\lambda+\alpha+\beta}{n}$.

In the special case, where $\alpha=0$ and $\beta=0$, system (7) reduces to

$$
\left\{\begin{array}{l}
u(x)=\int_{R^{n}} \frac{v^{q}(y)}{|x-y|^{\lambda}} d y \\
v(x)=\int_{R^{n}} \frac{u^{p}(y)}{|x-y|^{\lambda}} d y
\end{array}\right.
$$

with

$$
\frac{1}{q+1}+\frac{1}{p+1}=\frac{\lambda}{n}
$$

This integral system is closely related to the system of partial differential equations

$$
\left\{\begin{array}{l}
(-\Delta)^{\gamma / 2} u=v^{q}, u>0, \text { in } R^{n} \\
(-\Delta)^{\gamma / 2} v=u^{p}, \quad v>0, \text { in } R^{n}
\end{array}\right.
$$

where $\gamma=n-\lambda$.

In the special case when $p=q=\frac{n+\gamma}{n-\gamma}$, and $u(x)=v(x)$, system (8) becomes the single equation

$$
u(x)=\int_{R^{n}} \frac{u(y)^{\frac{n+\gamma}{n-\gamma}}}{|x-y|^{n-\gamma}} d y, \quad u>0, \text { in } R^{n} .
$$

The corresponding PDE is the well-known family of semilinear equations

$$
(-\Delta)^{\gamma / 2} u=u^{(n+\gamma) /(n-\gamma)}, u>0, \quad \text { in } R^{n} .
$$

In particular, when $n \geq 3$, and $\gamma=2$, (12) becomes

$$
-\Delta u=u^{(n+2) /(n-2)}, \quad u>0, \text { in } R^{n} .
$$

The classification of the solutions of (13) has provided an important ingredient in the study of the well-known Yamabe problem and the prescribing scalar curvature problem. It is also essential in deriving a priori estimates in many related nonlinear elliptic equations.

Equation (13) was studied by Gidas, Ni, and Nirenberg [7], Caffarelli, Gidas, and Spruck [1], Chen and Li [2], and Li [10]. They classified all the solutions. Recently, Wei and $\mathrm{Xu}$ [14] generalized this result to the solutions of the more general equation (12) with $\gamma$ being any even number between 0 and $n$.

Apparently, for other real values of $\gamma$ between 0 and $n$, equation (12) is also of practical interest and importance.

In [11], Lieb classified all the maximizers of the functional (5) under the constraints $\|f\|_{r}=1=\|g\|_{s}$ in the special case where $p=q=\frac{n+\gamma}{n-\gamma}$ and thus obtained 
the best constant in the HLS inequalities in that case. He then posed the classification of all the critical points of the functional and the solutions of the integral equation (11) as an open problem.

Chen, Li, and Ou solved this open problem in [4]. They proved:

Proposition 1. i) All solutions of the partial differential equation (12) satisfy the integral equation (11), and vice versa.

ii) If $u$ is a positive solution of (11) or (12) that is locally $L^{\frac{2 n}{n-\gamma}}\left(R^{n}\right)$, then u must be radially symmetric and decreasing about some point $x_{o}$ and therefore assumes the form of

$$
c\left(\frac{t}{t^{2}+\left|x-x_{o}\right|^{2}}\right)^{(n-\gamma) / 2}
$$

with some positive constants $c$ and $t$.

This proposition unifies and extends all the previous results on the family of partial differential equations (12).

Then in [5], Chen, Li, and Ou considered a more general system (8) and established the symmetry and monotonicity of the solutions.

Proposition 2. Let $(u, v)$ be a pair of solutions of (8) with $p, q \geq 1$. Assume that $u \in L^{p+1}\left(R^{n}\right)$ and $v \in L^{q+1}\left(R^{n}\right)$. Then $u$ and $v$ are radially symmetric and decreasing about some point $x_{o}$.

In [3], Chen and Li also obtained the regularity of the solutions.

Proposition 3. Assume that $(u(x), v(x))$ is a pair of positive solutions of (8), and $u \in L^{p+1}\left(R^{n}\right)$ and $v \in L^{q+1}\left(R^{n}\right)$. Then $u(x)$ and $v(x)$ are uniformly bounded in $R^{n}$, and therefore continuous and smooth.

To establish the symmetry of the solution, Chen, Li, and Ou ([4], [5], [6]) introduced a new idea, an integral form of the method of moving planes. It is entirely different from the traditional method used for partial differential equations. Instead of relying on maximum principles, certain integral norms were estimated. We believe that this new idea will become a powerful tool in studying qualitative properties of other integral equations and systems.

Following Chen, Li, and Ou's work, Jin and Li [8] studied the symmetry of the solutions to the more general system (7) and obtained

Proposition 4. Let the pair $(u, v)$ be a positive solution of system (7) with $u \in$ $L^{p+1}\left(R^{n}\right), v \in L^{q+1}\left(R^{n}\right), p, q \geq 1, p q \neq 1$, and $\alpha, \beta \geq 0$. Then $u$ and $v$ are radially symmetric and decreasing about some point $x_{o}$.

Jin and Li [9] also thoroughly discussed the regularity for the solutions to (7).

In this paper, based on the radial symmetry of the solutions established in [8], we will study the uniqueness of the solutions of the weighted integral system (7). We will start with the special case when $\gamma=2$, in which the integral system is equivalent to the nonlinear singular PDE system:

$$
\left\{\begin{array}{l}
-\triangle\left(|x|^{\alpha} u(x)\right)=\frac{v^{q}(x)}{|x|^{\beta}}, \\
-\triangle\left(|x|^{\beta} v(x)\right)=\frac{u^{p}(x)}{|x|^{\alpha}} .
\end{array}\right.
$$


It is obvious that the radial symmetry of the solutions reduces (15) to a system of ODEs, which has singularities at the origin. For a single equation, one can rather easily obtain uniqueness through some standard ODE arguments. However, for systems, there have not been such results. It is quite difficult to match well. Although the radial symmetry is a good starting point, an accurate estimate of the asymptotic behavior is the key.

Let

$$
f(x)=|x|^{\alpha} u(x), \quad g(x)=|x|^{\beta} v(x) .
$$

Then (15) becomes

$$
\left\{\begin{array}{l}
-\triangle f(x)=\frac{g^{q}(x)}{|x|^{\beta(q+1)}}, \\
-\triangle g(x)=\frac{f^{p}(x)}{|x|^{\alpha(p+1)}} .
\end{array}\right.
$$

Under the conditions

$$
\lambda+\beta(q+1)<n \quad \text { and } \quad \lambda+\alpha(p+1)<n,
$$

we prove

Theorem 1. The solutions of the system (16) are unique in the sense that if $\left(f_{1}, g_{1}\right)$ and $\left(f_{2}, g_{2}\right)$ are any two pairs of solutions with

$$
f_{1}(0)=f_{2}(0),
$$

then

$$
g_{1}(0)=g_{2}(0)
$$

and hence

$$
\left(f_{1}(x), g_{1}(x)\right) \equiv\left(f_{2}(x), g_{2}(x)\right) .
$$

This uniqueness theorem would enable one to classify all the solutions of the system (16) and hence obtain the best constant in the corresponding weighted Hardy-Littlewood-Sobolev inequality. At least, in some special cases, we are able to prove:

Theorem 2. Assume that

$$
\alpha=\beta=\frac{n}{p+1}-\frac{n-2}{2} \text { and } p=q .
$$

Let $(f, g)$ be any pair of solutions of system (16). Then $f(x)=g(x)$, and they must both assume the form of

$$
\phi_{t}(x)=c\left[\frac{t}{t^{2}+|x|^{\frac{(n-2)(p-1)}{2}}}\right]^{\frac{2}{p-1}}
$$

with some real number $t$ and some constant $c$.

On the other hand, for each real number $t$, there is a constant $c=c(t)$, such that $\left(\phi_{t}(x), \phi_{t}(x)\right)$ is a pair of solutions for (16).

We will prove these two theorems in the next section. One main ingredient in the proof of Theorem 1 is the asymptotic behavior of the solutions near the origin and at infinity. 


\section{Proof of the uniqueness}

The key to the proof of uniqueness is the asymptotic behavior of the solutions near the origin and at infinity. This was established in a paper of Li and Lim [12] as stated in the following proposition.

Proposition 2.1. Let $(u, v) \in L^{p+1}\left(R^{n}\right) \times L^{q+1}\left(R^{n}\right)$ be a pair of positive solutions of the system (7). Suppose that $p, q \geq 1, p q \neq 1$, and $\alpha+\beta \geq 0$.

(i) If $\lambda+\beta(q+1)<n$, then for small $|x|$, we have

$$
u(x) \simeq \frac{A_{o}}{|x|^{\alpha}}
$$

and

$$
v(x) \simeq \begin{cases}\frac{A_{1}}{|x|^{\beta},} & \text { if } \lambda+\alpha(p+1)<n, \\ \frac{A_{2}}{|x|^{\alpha(p+1)+\beta+\lambda-n},}, & \text { if } \lambda+\alpha(p+1)>n, \\ \frac{A_{3}|\ln | x||}{|x|^{\beta}}, & \text { if } \lambda+\alpha(p+1)=n,\end{cases}
$$

where

$$
\begin{aligned}
& A_{o}=\int_{R^{n}} \frac{v^{q}(y)}{|y|^{\lambda+\beta}} d y, \quad A_{1}=\int_{R^{n}} \frac{u^{p}(y)}{|y|^{\lambda+\alpha}} d y, \\
& A_{2}=\left(\int_{R^{n}} \frac{v^{q}(y)}{|y|^{\lambda+\beta}} d y\right)^{p} \int_{R^{n}} \frac{d z}{|z|^{\alpha(p+1)}|e-z|^{\lambda}}, \\
& A_{3}=\left|S^{n-1}\right|\left(\int_{R^{n}} \frac{v^{q}(y)}{|y|^{\lambda+\beta}} d y\right)^{p} .
\end{aligned}
$$

Here $e$ is a unit vector in $R^{n}$ and $\left|S^{n-1}\right|$ is the surface area of the unit sphere.

(ii) If $\lambda q+\beta(q+1)>n$, then for large $|x|$, we have

$$
u(x) \simeq \frac{B_{o}}{|x|^{\lambda+\alpha}}
$$

and

$$
v(x) \simeq \begin{cases}\frac{B_{1}}{|x|^{\lambda+\beta}}, & \text { if } \lambda p+\alpha(p+1)>n, \\ \frac{|x|_{2}^{(\alpha+\lambda)(p+1)+\beta-n}}{\mid(\ln |x| \mid}, & \text { if } \lambda p+\alpha(p+1)<n, \\ \frac{B_{3}|x|^{\lambda+\beta}}{\mid \lambda+\beta} & \text { if } \lambda p+\alpha(p+1)=n,\end{cases}
$$

where

$$
\begin{aligned}
& B_{o}=\int_{R^{n}} \frac{v^{q}(y)}{|y|^{\beta}} d y, \quad B_{1}=\int_{R^{n}} \frac{u^{p}(y)}{|y|^{\alpha}} d y, \\
& B_{2}=\left(\int_{R^{n}} \frac{v^{q}(y)}{|y|^{\beta}} d y\right) \int_{R^{n}} \frac{d z}{|z|^{2 n-(\alpha+\lambda)(p+1)}|e-z|^{\lambda}},
\end{aligned}
$$

and

$$
B_{3}=\left|S^{n-1}\right|\left(\int_{R^{n}} \frac{v^{q}(y)}{|y|^{\beta}} d y\right)^{p} .
$$

The Proof of Theorem 1. Let $(f, g)$ be a pair of solutions to system (16).

It follows from condition (17) and Proposition 2.1 that

$$
\lim _{x \rightarrow 0} f(x)=A_{o}, \quad \lim _{x \rightarrow 0} g(x)=A_{1} .
$$


Again by Proposition 2.1, we obtain, in all cases, the asymptotic behaviors of the solutions at infinity:

$$
f(x) \rightarrow 0 \text { and } g(x) \rightarrow 0, \quad \text { as } x \rightarrow \infty .
$$

By virtue of Proposition 4, the solutions of (16) are radially symmetric; hence we can write, in polar coordinates, the first equation in (16) as

$$
\left(r^{n-1} f^{\prime}(r)\right)^{\prime}=-r^{n-1-\beta(q+1)} g^{q}(r),
$$

where $r=|x|$.

Integrating both sides from 0 to $r$ yields

$$
r^{n-1} f^{\prime}(r)=-\int_{0}^{r} s^{n-1-\beta(q+1)} g^{q}(s) d s .
$$

It follows by another integration that

$$
f(r)=f(0)-\int_{0}^{r} \frac{1}{\tau^{n-1}} \int_{0}^{\tau} s^{n-1-\beta(q+1)} g^{q}(s) d s d \tau .
$$

Similarly, for $g(r)$, we have

$$
g(r)=g(0)-\int_{0}^{r} \frac{1}{\tau^{n-1}} \int_{0}^{\tau} s^{n-1-\alpha(p+1)} f^{p}(s) d s d \tau .
$$

Let $\left(f_{1}(x), g_{1}(x)\right)$ and $\left(f_{2}(x), g_{2}(x)\right)$ be any two pairs of solutions of (16). Assume that $f_{1}(0)=f_{2}(0)$. We are going to show that $g_{1}(0)=g_{2}(0)$. Otherwise, suppose

$$
g_{1}(0)<g_{2}(0) \text {. }
$$

Then by continuity, for small $r$,

$$
g_{1}(r)<g_{2}(r) .
$$

Now applying (25) to both $\left(f_{1}(x), g_{1}(x)\right)$ and $\left(f_{2}(x), g_{2}(x)\right)$, and by $(28)$, we see that there exists an $R>0$ such that

$$
f_{1}(r)>f_{2}(r) \text { and } g_{1}(r)<g_{2}(r), \forall r \in(0, R) .
$$

Let $R_{o}$ be the supreme value of $R$ such that (29) holds.

i) If $R_{o}=\infty$, then from (24), we must have

$$
f_{1}(r) \rightarrow 0, f_{2}(r) \rightarrow 0, \quad \text { as } r \rightarrow \infty .
$$

Hence it follows from (25) and the assumption $f_{1}(0)=f_{2}(0)$ that

$$
\int_{0}^{\infty} \frac{1}{\tau^{n-1}} \int_{0}^{\tau} s^{n-1-\beta(q+1)} g_{1}^{q}(s) d s d r=\int_{0}^{\infty} \frac{1}{\tau^{n-1}} \int_{0}^{\tau} s^{n-1-\beta(q+1)} g_{2}^{q}(s) d s d r .
$$

This is impossible due to the second part of (29).

ii) If $R_{o}$ is finite, then we have

$$
f_{1}(r)>f_{2}(r) \text { and } g_{1}(r)<g_{2}(r), \quad \forall r \in\left(0, R_{o}\right),
$$

while either

$$
f_{1}\left(R_{o}\right)=f_{2}\left(R_{o}\right)
$$

or

or both

$$
g_{1}\left(R_{o}\right)=g_{2}\left(R_{o}\right),
$$

$$
f_{1}\left(R_{o}\right)=f_{2}\left(R_{o}\right) \text { and } g_{1}\left(R_{o}\right)=g_{2}\left(R_{o}\right) .
$$


These will again contradict (25) and (26), more precisely, contradict

$$
f_{i}\left(R_{o}\right)=f_{i}(0)-\int_{0}^{R_{o}} \frac{1}{\tau^{n-1}} \int_{0}^{\tau} s^{n-1-\beta(q+1)} g_{i}^{q}(s) d s d \tau, \quad i=1,2,
$$

and

$$
g_{i}\left(R_{o}\right)=g_{i}(0)-\int_{0}^{R_{o}} \frac{1}{\tau^{n-1}} \int_{0}^{\tau} s^{n-1-\alpha(p+1)} f_{i}^{p}(s) d s d \tau, \quad i=1,2 .
$$

In fact, say, in the case $g_{1}\left(R_{o}\right)=g_{2}\left(R_{o}\right)$, then by (31), we have

$$
\begin{aligned}
& g_{1}(0)-\int_{0}^{R_{o}} \frac{1}{\tau^{n-1}} \int_{0}^{\tau} s^{n-1-\alpha(p+1)} f_{1}^{p}(s) d s d \tau \\
& \quad=g_{2}(0)-\int_{0}^{R_{o}} \frac{1}{\tau^{n-1}} \int_{0}^{\tau} s^{n-1-\alpha(p+1)} f_{2}^{p}(s) d s d \tau .
\end{aligned}
$$

This, together with the assumption $g_{1}(0)<g_{2}(0)$, implies

$$
\int_{0}^{R_{o}} \frac{1}{\tau^{n-1}} \int_{0}^{\tau} s^{n-1-\alpha(p+1)} f_{1}^{p}(s) d s d \tau<\int_{0}^{R_{o}} \frac{1}{\tau^{n-1}} \int_{0}^{\tau} s^{n-1-\alpha(p+1)} f_{2}^{p}(s) d s d \tau .
$$

This is impossible due to the first part of (30). Similarly, one can derive contradictions in the other remaining cases.

Hence it is impossible that $g_{1}(0)<g_{2}(0)$. Similarly, one can show that $g_{1}(0)>$ $g_{2}(0)$ is impossible. Therefore, we must have

$$
g_{1}(0)=g_{2}(0)
$$

Finally, by the standard ODE theory, we arrive at

$$
\left(f_{1}(x), g_{1}(x)\right) \equiv\left(f_{2}(x), g_{2}(x)\right) .
$$

This completes the proof of Theorem 1.

The Proof of Theorem 2. On the one hand, by a straightforward calculation, one can verify that for each real number $t$, there is a constant $c=c(t)$, such that $\left(\phi_{t}(x), \phi_{t}(x)\right)$ defined by (18) is a pair of solutions for (16).

On the other hand, assume that $(f(x), g(x))$ is a pair of solutions of (16). Choose real numbers $t$ and $c$ such that

$$
\phi_{t}(0)=f(0)
$$

Then, for such a value of $t$, since $\left(\phi_{t}(x), \phi_{t}(x)\right)$ is a pair of solutions of $(16)$, by the uniqueness result of Theorem 1, we must have

$$
g(0)=\phi_{t}(0)
$$

and therefore

$$
(f(x), g(x)) \equiv\left(\phi_{t}(x), \phi_{t}(x)\right) .
$$

This completes the proof of the theorem. 


\section{REFERENCES}

[1] L. Caffarelli, B. Gidas, and J. Spruck, Asymptotic symmetry and local behavior of semilinear elliptic equations with critical Sobolev growth, Comm. Pure Appl. Math. XLII, (1989), 271297. MR982351 (90c:35075)

[2] W. Chen and C. Li, Classification of solutions of some nonlinear elliptic equations, Duke Math. J., 63 (1991), 615-622. MR1121147 (93e:35009)

[3] W. Chen and C. Li, Regularity of Solutions for a system of Integral Equations, Comm. Pure and Appl. Anal., 4(2005), 1-8. MR2126275 (2006g:45006)

[4] W. Chen, C. Li, and B. Ou, Classification of solutions for an integral equation, Comm. Pure and Appl. Math., 59(2006), 330-343. MR2200258 (2006m:45007a)

[5] W. Chen, C. Li, and B. Ou, Classification of solutions for a system of integral equations, Comm. in Partial Differential Equations, 30(2005), 59-65. MR2131045 (2006a:45007)

[6] W. Chen, C. Li, and B. Ou, Qualitative Properties of Solutions for an Integral Equation, Disc. \& Cont. Dynamics Sys., 12(2005), 347-354. MR2122171 (2006g:45009)

[7] B. Gidas, W. M. Ni, and L. Nirenberg, Symmetry of positive solutions of nonlinear elliptic equations in $R^{n}$, Mathematical Analysis and Applications, Academic Press, New York, 1981. MR634248 (84a:35083)

[8] C. Jin and C. Li, Symmetry of Solutions to Some Integral Equations, Proc. Amer. Math. Soc., 134(2006), 1661-1670. MR2204277 (2006j:45017)

[9] C. Jin and C. Li, Quantitative Analysis of Some System of Integral Equations, Cal. Var. PDEs, 26(2006), 447-457. MR2235882 (2007c:45013)

[10] C. Li, Local asymptotic symmetry of singular solutions to nonlinear elliptic equations, Invent. Math., 123 (1996), 221-231. MR1374197 (96m:35085)

[11] E. Lieb, Sharp constants in the Hardy-Littlewood-Sobolev and related inequalities, Ann. of Math., 118(1983), 349-374. MR717827 (86i:42010)

[12] C. Li and J. Lim, The singularity analysis of solutions to some integral equations, Commun. Pure Appl. Anal. 6 (2007), 453-464. MR2289831

[13] E. M. Stein and G. Weiss, Fractional integrals in $n$-dimensional Euclidean space J. Math. Mech., 7 (1958). MR0098285 (20:4746)

[14] J. Wei and X. Xu, Classification of solutions of higher order conformally invariant equations, Math. Ann., 313 (1999), 207-228. MR1679783 (2000a:58093)

College of Mathematics and Information Science, Henan Normal University, PeoPLE's RePUBlic OF ChinA

Current address: Department of Mathematics, Yeshiva University, 500 W. 185th Street, New York, New York 10033

E-mail address: wchen@yu.edu

Department of Applied Mathematics, University of Colorado, Boulder, Colorado 80309

E-mail address: cli@colorado.edu 